\title{
Application of electrophysiological measures in spinal cord injury clinical trials: a narrative review
}

\author{
Michèle Hubli $^{1}$ - John L. K. Kramer ${ }^{2,3} \cdot$ Catherine R. Jutzeler $^{1,2,4} \cdot$ Jan Rosner $^{1,5}$ • Julio C. Furlan ${ }^{6,7}$. \\ Keith E. Tansey ${ }^{8,9,10} \cdot$ Martin Schubert $^{1}$
}

Received: 12 February 2019 / Revised: 8 July 2019 / Accepted: 9 July 2019 / Published online: 23 July 2019

(c) The Author(s), under exclusive licence to International Spinal Cord Society 2019

\begin{abstract}
Study design Narrative review.

Objectives To discuss how electrophysiology may contribute to future clinical trials in spinal cord injury (SCI) in terms of: (1) improvement of SCI diagnosis, patient stratification and determination of exclusion criteria; (2) the assessment of adverse events; and (3) detection of therapeutic effects following an intervention.

Methods An international expert panel for electrophysiological measures in SCI searched and discussed the literature focused on the topic.

Results Electrophysiology represents a valid method to detect, track, and quantify readouts of nerve functions including signal conduction, e.g., evoked potentials testing long spinal tracts, and neural processing, e.g., reflex testing. Furthermore, electrophysiological measures can predict functional outcomes and thereby guide rehabilitation programs and therapeutic interventions for clinical studies.

Conclusion Objective and quantitative measures of sensory, motor, and autonomic function based on electrophysiological techniques are promising tools to inform and improve future SCI trials. Complementing clinical outcome measures, electrophysiological recordings can improve the SCI diagnosis and patient stratification, as well as the detection of both beneficial and adverse events. Specifically composed electrophysiological measures can be used to characterize the topography and completeness of SCI and reveal neuronal integrity below the lesion, a prerequisite for the success of any interventional trial. Further validation of electrophysiological tools with regard to their validity, reliability, and sensitivity are needed in order to become routinely applied in clinical SCI trials.
\end{abstract}

\section{Introduction}

In 2007, an international panel supported by multiple foundations, under the banner International Campaign for

Michèle Hubli

Michele.Hubli@balgrist.ch

1 Spinal Cord Injury Center, Balgrist University Hospital, University of Zurich, Zurich, Switzerland

2 International Collaboration on Repair Discoveries (ICORD), University of British Columbia, Vancouver, BC, Canada

3 School of Kinesiology, University of British Columbia, Vancouver, BC, Canada

4 Department of Biosystems Science and Engineering, ETH Zurich, Basel, Switzerland

5 Department of Neurology, Inselspital, Bern University Hospital,
Cures of Spinal Cord Injury Paralysis, published an initial set of papers [1-4] reviewing spinal cord injury (SCI) clinical trial methodology, as well as providing recommendations for future studies. After a decade, the SCI 
research community acknowledges some persistent shortcomings and barriers to translational research success, as well as new challenges and opportunities for the conduct of studies. The Spinal Trials Understanding, Design, and Implementation (STUDI) initiative was established by several SCI-focussed foundations (see sponsorship) in 2016 to report on better management of participant recruitment and study protocols, while emphasizing additional approaches to more effectively select and track outcomes in clinical trials, including electrophysiology, imaging, and biomarker tools. STUDI launches the development of a new interactive website (scitrials.org) that continually updates information about ongoing studies and facilitates participation from people living with SCI.

Clinical trials on therapeutic interventions for the neurological restoration of SCI require improved assessment techniques and quantitative outcome measures to complement and overcome the limitations as experienced by solely relying on the International Standards for Neurological Classification of Spinal Cord Injury (ISNCSCI) $[2,5,6]$ and the International Standards to document remaining Autonomic Function after Spinal Cord Injury (ISAFSCI) [7]. More objective outcome measures will be an essential step forward in the implementation of interventional trials, in which proof-of-concept is currently still limited to ordinal clinical readouts. Stratification of highly diverse study participants into homogenous clinical trial cohorts is an important prerequisite for clinical trial success [8]. Adequate outcome assessments and sensitive biomarkers are not only needed as surrogate indicators of safety of experimental therapeutics, but also as critical endpoints of clinical efficacy [9].

While clinical assessments (e.g., ISNCSCI, ISAFSCI, and behavioral outcomes testing) provide a general evaluation of the sensorimotor function, electrophysiological methods reveal neuroanatomical, and physiological assessments of the peripheral and central nervous system improving functional prediction [10]. For example, using the American Spinal Injury Association Impairment Scale (AIS) as primary outcome for a clinical SCI trials is problematic since a considerable amount of conversions in AIS grade might not reflect a change in severity of neurological deficit, but is rather heavily dependent on the scoring of sacral segments [11]. Here, electrophysiological measures might be advantageous improving the assessment of lesion completeness [12-15]. Furthermore, they could improve our understanding of mechanisms of action of therapeutic interventions with regards to neural plasticity, axonal growth, and/or remyelination as they can be used to assess the conduction and processing of neural signals.

In general, within the last few decades much promise has been given to electrophysiology as a measure to assess and stratify SCI, predict functional outcomes, and inform clinicians about the planning and results of therapeutic interventions. In this regard one should also review the Common Data Elements recently developed by the National Institutes of Health in the USA [16]. While these Common Data Elements describe a broad spectrum of electrophysiological examination methods in detail, only a portion of them will be included here. Mainly routinely applied electrophysiological measures that are available in the clinical neurological setting were chosen (for an overview see Table 1), since many clinical sites do not have access and expertise to perform highly experimental electrophysiological test batteries. This current review will provide an improved understanding for electrophysiological outcome assessment use in SCI clinical trials. After a first section on how clinical assessments can be complemented with electrophysiological readouts, more insights and illustrative examples will be given in order to showcase how electrophysiological recordings can: (1) improve the SCI diagnosis and contribute to determination of exclusion criteria and patient stratification for SCI trials; (2) facilitate the assessment of adverse events during an SCI trial; (3) enable the potential detection of even subclinical biological effects after therapeutic interventions.

\section{Electrophysiology to complement clinical assessments}

Following a traumatic lesion of the spinal cord, clinical examination is usually the first and most important diagnostic tool. Delineating the level and severity of lesion is currently done using the ISNCSCI [17] and the ISAFSCI [7], which are well accepted clinical measures of sensorimotor and autonomic function in the SCI population. While these clinical assessments are pivotal to acquire an initial status of the injury, they lack the sensitivity to provide detailed information about the damage to the individual sensory, motor and autonomic pathways. Is the impairment a result of upper motor neuron (UMN) or lower motor neuron (LMN) damage or a combination? Does the patient report hyper- or hypoalgesia? The AIS reports neurological level of injury and gross sensory and motor deficits with an emphasis on rectal sensorimotor function, but neglects the distribution and extent of residual functions. Moreover, there is currently no assessment of positive phenomena, such as increased muscle tone (hyperreflexia, spasticity), nor of processing of neural signals across and below the level of lesion.

Overcoming some limitations of conventional clinical assessments, electrophysiology is a set of valid tools that examine across an array of physiological mechanisms to detect, track, and quantify readouts of biological signal transmission and processing. In this regard 


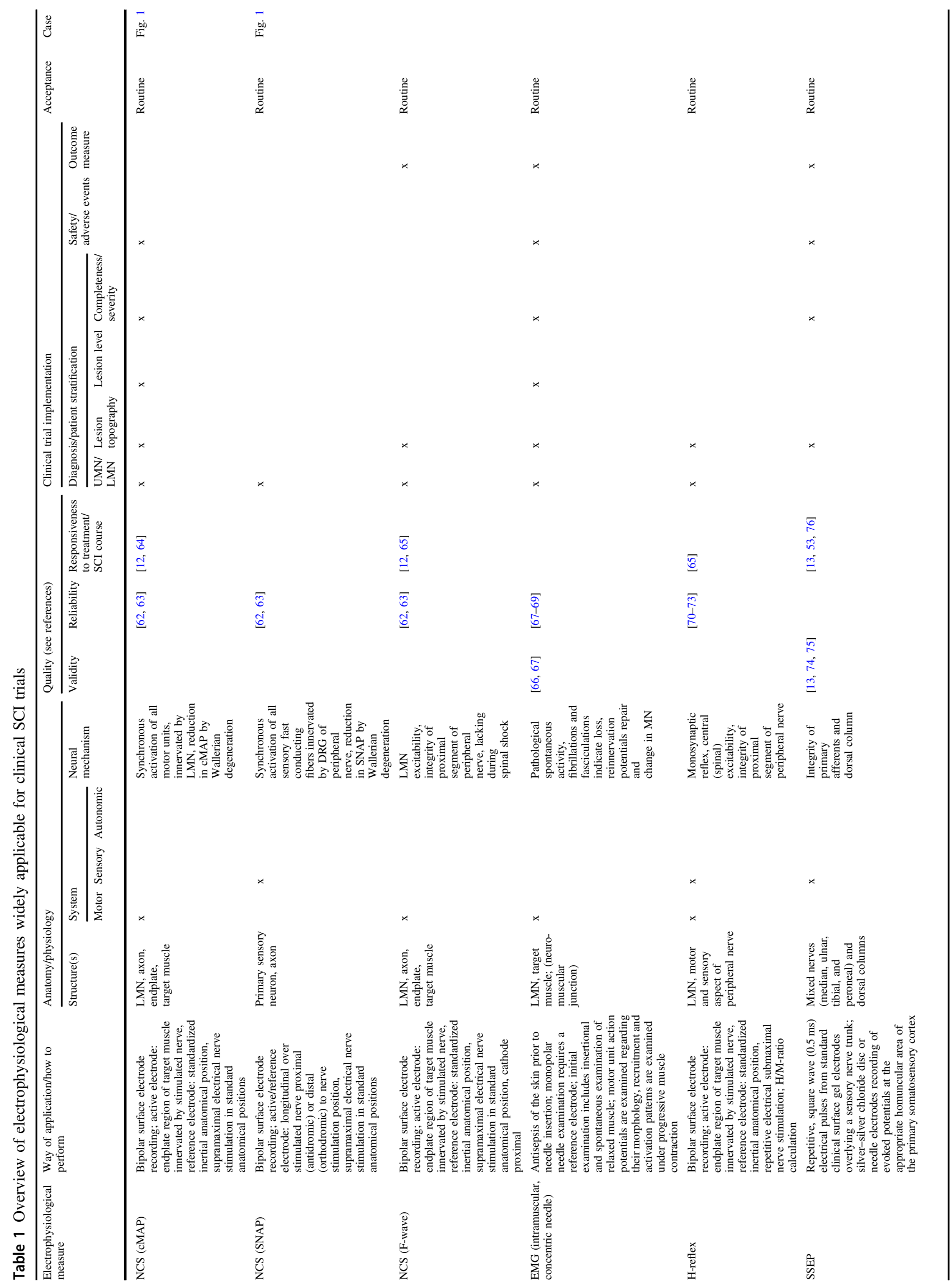




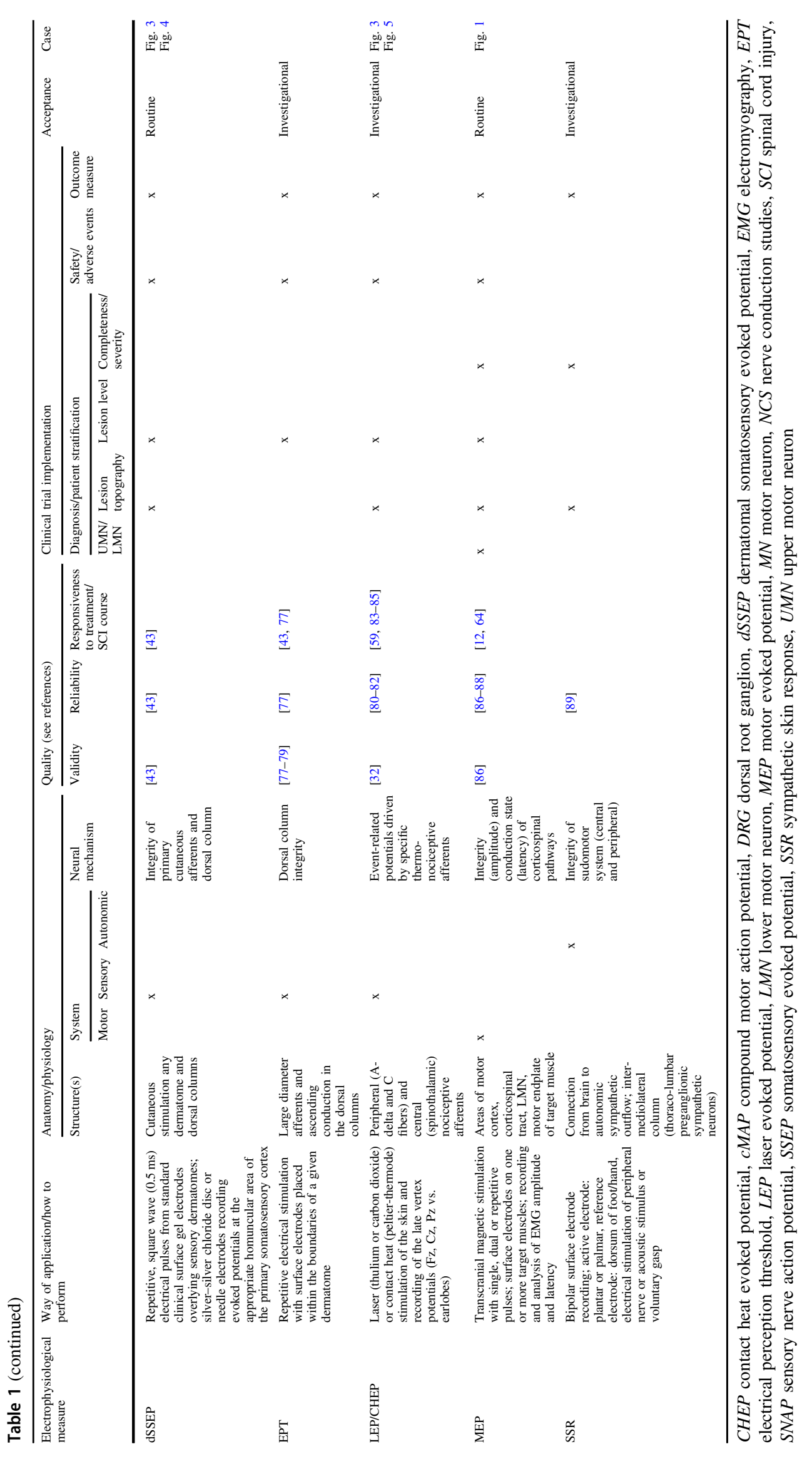


electrophysiology can be advantageous for a number of reasons: (1) SCI profiling for exclusion/inclusion/stratification: electrophysiological tests can help to rule out unrecognized diseases of the peripheral nervous system that may precede SCI (i.e., diabetic polyneuropathy, nerve entrapment syndromes) or identify concomitant disorders (i.e., critical illness polyneuropathy and myopathy, or later pressure neuropathies, traumatic nerve, or plexus injury), which may adversely affect motor, sensory, and autonomic recovery and flaw the enrolment of appropriate patients. (2) Objectivity and scalability: electrophysiological recordings provide objective and quantitative data that can be analyzed by blinded researchers. The readouts are interval-scaled or ratio-scaled and thus less arbitrary than the more subjective clinical assessments. (3) Applicability and stability: electrophysiological recordings can also be attained in unresponsive, uncooperative or comatose patients, which can be valuable for acute diagnosis. (4) Sensitivity: electrophysiological measures can be more sensitive to detect subclinical changes (i.e., those that may not be detectable by a clinical assessment), but could guide changes in therapeutic dose and/or administration protocol [18]. (5) Pathophysiology of the nervous system: electrophysiological tests can facilitate our understanding of the underlying pathophysiological mechanisms of SCI and how the neurological recovery is modified by therapeutic interventions.

\section{$\mathrm{SCl}$ diagnosis and patient stratification}

Electrophysiological protocols can be tailored to improve the assessment of the cord damage, such as topography of affected longitudinal spinal pathways (e.g., impairment of the dorsal column-medial lemniscal, spinothalamic, and/or motor pathways) and diagnosis of typical spinal syndromes (e.g., anterior cord, central cord, and Brown-Séquard syndrome). Furthermore, the rostrocaudal extent of the lesion can be assessed by segmental tests, such as dermatomal somatosensory evoked potentials (dSSEPs) and contact heat evoked potentials (CHEPs), as well as electromyography (EMG) and motor nerve conduction studies (NCS). Although technically demanding, electrophysiology is immediately assessing neurological function. In concert with clinical exams, neuroimaging, behavioral testing, and patient reported outcomes, electrophysiology tremendously improves our understanding of underlying pathophysiology thereby guiding and assisting patient stratification in a clinical SCI trial. In the following four sections the merit of electrophysiology is emphasized with regard to improved assessment/diagnosis of: (1) UMN and LMN lesion, (2) lesion topography (axial distribution), (3) lesion level and rostrocaudal extent, and (4) lesion severity.

\section{SCI - upper and lower motor neuron lesions}

Traumatic SCI inherently represents the combined damage of both segmental central and peripheral neural structures, in particular of UMN and LMN, respectively at the lesion zone. Preserved function of motor neurons and neuronal circuits below and across the level of the lesion is one prerequisite for successful rehabilitation training and for interventions aiming at structural neuroregeneration. It is therefore necessary to determine the extent of the spinal injury zone and whether the spinal cord below the lesion has some preserved functional connections [19].

Myelomalacia below the spinal lesion may occur due to spinal ischemia secondary to the trauma. It is known to be characterized by permanent loss of tendon reflexes and muscle tone and it affects motor NCS, i.e., compound motor action potentials (CMAP) and F-waves [20, 21]. Furthermore, several studies revealed electrophysiological evidence of damage to the spinal (lower) motor neurons far below the lesion zone by employing CMAPs, F-wave persistence and axon excitability studies, such as threshold tracking [12, 22-24]. This damage to the LMN far below the lesion zone is not due to a local injury inflicted at the spinal trauma zone but rather a secondary deterioration within the peripheral motor system depending on anatomical distance caudal to lesion and on lesion severity [12]. Decentralization, i.e., loss of supraspinal motor control, and the consequent immobility are likely to underlie these complex changes observed in axonal excitability below the lesion in SCI patients [24]. Therefore, testing of reflex activity (tendon and H-Reflex), and of spinal excitability and integrity of the LMN may be assessed below the lesion in SCI clinical trials.

Peripheral NCS and intramuscular EMG are also necessary to detect and characterize concomitant traumatic damage to the peripheral nervous system if present, such as radiculopathy, nerve plexus damage or peripheral neuropathy, and help distinguish them from spinal cord damage. NCS and EMG test the motor final common path of the LMN and in combination with sensory NCS allow for differentiation of muscle paresis due to damage of spinal anterior (ventral) horn cell (LMN), anterior nerve root lesions or peripheral nerve damage (plexus, peripheral nerve) [25]. NCS and intramuscular EMG should be obtained in spinal segments prone to have undergone LMN damage: for cervical lesion median/ulnar nerve NCS and intramuscular EMG of target muscles innervated by cervical spinal segments [19, 26], for low thoracic/lumbar lesions peroneal/tibial nerve NCS and intramuscular EMG of target muscles innervated by lumbar/sacral spinal segments [22, 27, 28].

Furthermore, H-reflex and F-wave recordings not only prove integrity of the proximal segment of the peripheral 
nerve but also assess the excitability of LMN below the lesion [12]. In this regard, a posterior root injury or sensory neuropathy might lead to absent $\mathrm{H}$-reflexes in the presence of intact CMAP and F-wave, indicating intact LMN function in combination with segmental sensory deficits and/or peripheral nerve damage. An example of a tetraplegic patient with UMN and LMN damage and the concurrent electrophysiological measures is provided in Fig. 1.

\section{Summary and Considerations: The characterization of neural networks below the level of the lesion might be helpful to improve detection of exclusion criteria. Verification of integrity of the spinal cord caudal to the lesion and exclusion of peripheral nerve damage is necessary before including patients into clinical trials.}

Example: A chronic thoracic AIS A patient presenting with muscular atrophy and hypo-/areflexia below the lesion level should additionally be tested with NCS of the legs and H-reflex testing. If these also show no responses (i.e., complete loss of CMAP and H-reflex) exclusion from a trial of neuroregeneration or epidural stimulation must ensue as myelomalacia is assumed. However, if the neurophysiological tests prove presence of CMAP and H-reflex and a lack of pathology in EMG, the clinical findings and the low reflex levels may be interpreted as a physiological variant of SCI or persistence of a degree of spinal shock.

\section{Topography of lesion/spinal cord syndromes}

Lesion topography, i.e., axial distribution, and, as a result, clinical spinal cord syndromes are relevant as they show distinct patterns of recovery related to the anatomy of spinal cord pathways [29]. In general, the ISNCSCI exam can only broadly suggest spinal tract deficits, while electrophysiological assessments are more precise by testing specific ascending and descending spinal cord pathways (see Fig. 2). SSEPs, CHEPs, and MEPs are used for testing conduction in long spinal tracts conveying afferent somatosensory and efferent motor information across the lesion site. SSEPs are known to test dorsal column function, while CHEPs or laser evoked potentials (LEPs) test spinothalamic pathways [30]. Detection of SSEPs confirms synchronous signal transmission through large diameter myelinated fibers in the periphery and within the ascending spinal dorsal columns. With additional recordings from the neck or

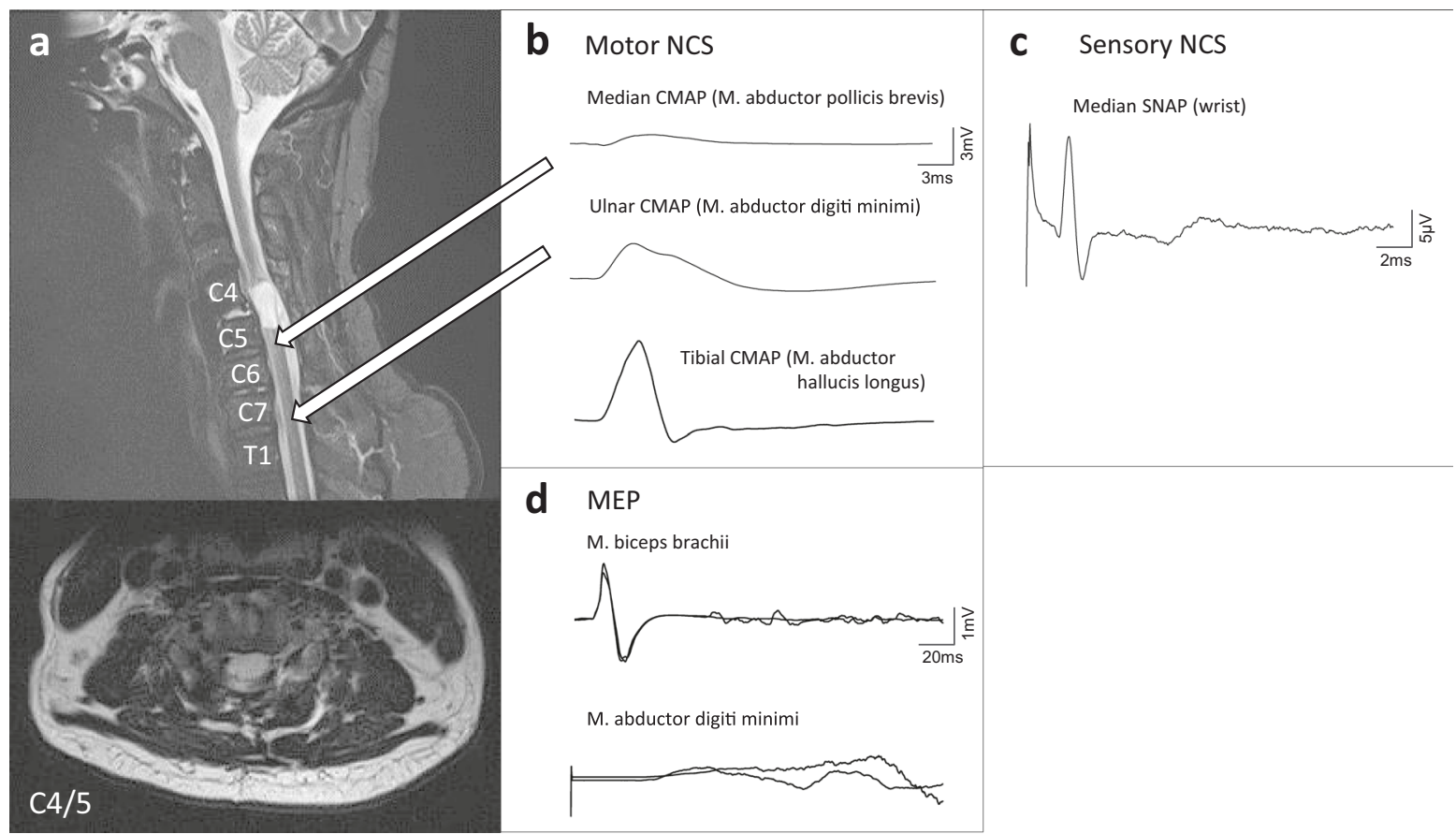

Fig. 1 Upper and lower motor neuron lesion. This patient presents with an incomplete (AIS B) traumatic SCI with a neurological level of lesion at C5 (T2-weighted sagittal and axial MRI of the cord (a)). Nerve conduction studies show a characteristic pattern: Motor NCS reveal strongly diminished median CMAP $(0.4 \mathrm{mV})$, but only minimal amplitude reduction of ulnar $(2.8 \mathrm{mV})$ and normal tibial CMAPs $(6.4$ $\mathrm{mV}$ ) are found below lesion level (b). Therefore no myelomalacia can be assumed in this case. LMN damage at lesion level is indicated by loss of median CMAP, but intact sensory nerve function is highlighted by intact median CSAP $(15.8 \mu \mathrm{V})(\mathbf{c})$. Next to the LMN, also UMN tract fibers projecting to $\mathrm{C} 8 / \mathrm{T} 1$ level are severely lesioned as is reflected in absent MEPs of the abductor digiti minimi, whereas MEPs of biceps brachii (C5/C6 level) is normally preserved (d). CMAP compound motor action potential, MEP motor evoked potential, NCS nerve conduction study, SNAP sensory nerve action potential 


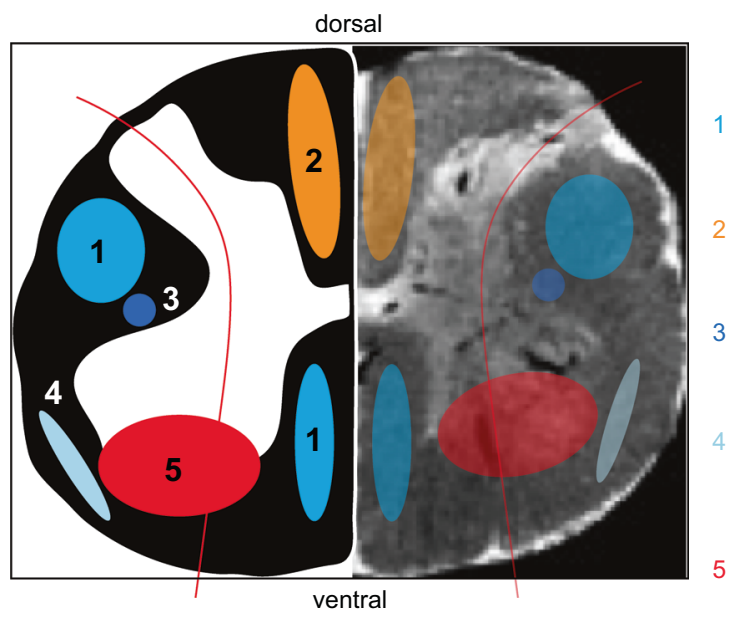

Fig. 2 Overview of electrophysiological techniques applicable to target specific spinal cord tracts and segments. Signal conduction in spinal tracts will be assessed with sensory and motor evoked potentials, as well as autonomic responses (i.e., SSR). Segmental function can be tested by dSSEPs, choice of target muscle in performing MEPs and choice of peripheral nerve, muscle or skin area in performing

$\begin{array}{ccc}\begin{array}{c}\text { SPINAL } \\ \text { PATHWAYS }\end{array} & \text { METHODS } & \begin{array}{c}\text { CLINICAL } \\ \text { CORRELATE }\end{array} \\ \text { corticospinal } & \text { TMS, MEP } & \text { central paresis } \\ \text { dorsal column } & \begin{array}{c}\text { EPT, SSEP, } \\ \text { dSSEP }\end{array} & \begin{array}{c}\text { cutaneous touch, } \\ \text { proprioception }\end{array} \\ \text { sympathetic } & \text { SSR } & \begin{array}{c}\text { sudomotor, cardiac, } \\ \text { vasomotor control }\end{array} \\ \text { spinothalamic } & \text { CHEP, LEP } & \begin{array}{c}\text { pain/temp } \\ \text { perception }\end{array} \\ & \text { NCS/Reflex } & \text { peripheral } \\ \text { paresis }\end{array}$

NCS/reflex testing, EMG and SSR. Numbers in the table refer to spinal tracts in the schematic drawing. CHEP contact heat evoked potential, dSSEP dermatomal somatosensory evoked potential, EMG electromyography, EPT electrical perception threshold, MEP motor evoked potential, NCS nerve conduction study, SSEP somatosensory evoked potential, SSR sympathetic skin response (adapted from [90])

the motor cortex. MEPs depend on direct corticospinal and indirect descending tracts such as reticulospinal and propriospinal pathways [34, 35]. Detection of an MEP confirms the functional integrity of the central innervation to the targeted muscle with the amplitude and latency of the MEP response providing some information about any central conduction impairment (e.g., by slowing of central motor conduction time) [36]. Crucially, MEPs of limb muscles reflect cortical as well as spinal/peripheral motor excitability and on its own cannot be used to distinguish damage at either location.

Dissociated loss of one somatosensory modality points toward a focal rather than diffuse spinal damage due to the topographical organization of afferent spinal tracts (Fig. 2). Specific spinal syndromes or damage patterns, with distinct potentials for recovery, can already be distinguished clinically. The ACS results in predominant damage of the ventral and lateral cord (the perfusion territory of the anterior spinal artery), according to Schneider [37] affecting the anterior two-thirds of the cord, including the segmental ventral horn cells, spinothalamic, and long motor tracts. The Brown-Séquard syndrome, due to a hemisection or lateralized lesion of the cord, presents with ipsilateral ataxia and paresis due to proprioceptive and motor loss in association with contralateral loss of pain and temperature sensation below the level of lesion. In both syndromes, damage within the spinothalamic system can be documented using noxious heat stimulation with evoked potential recordings [38] and related clinical pinprick testing. In traumatic central cord syndrome (CCS), the most common acute incomplete cervical SCI accounting for $44 \%$ of all spinal syndromes and 


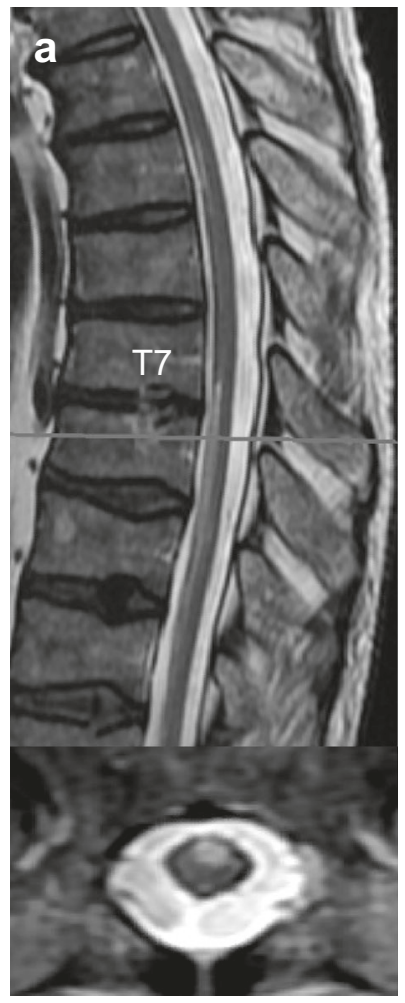

b

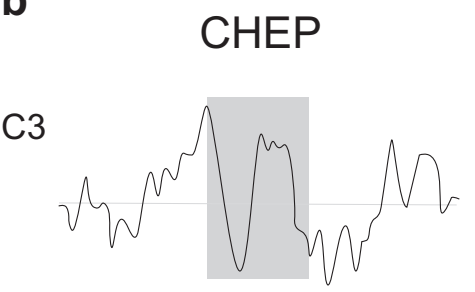

T7

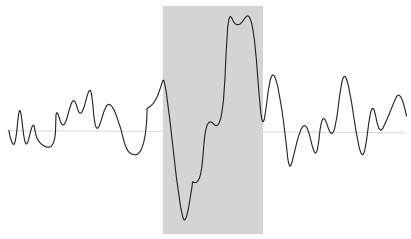

$\mathrm{T} 10$

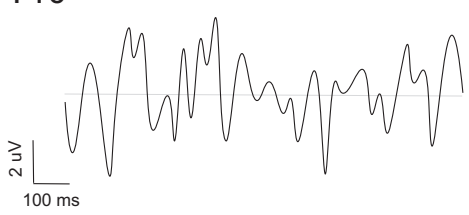

C

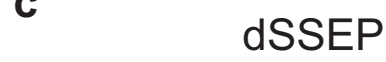

C3

T7
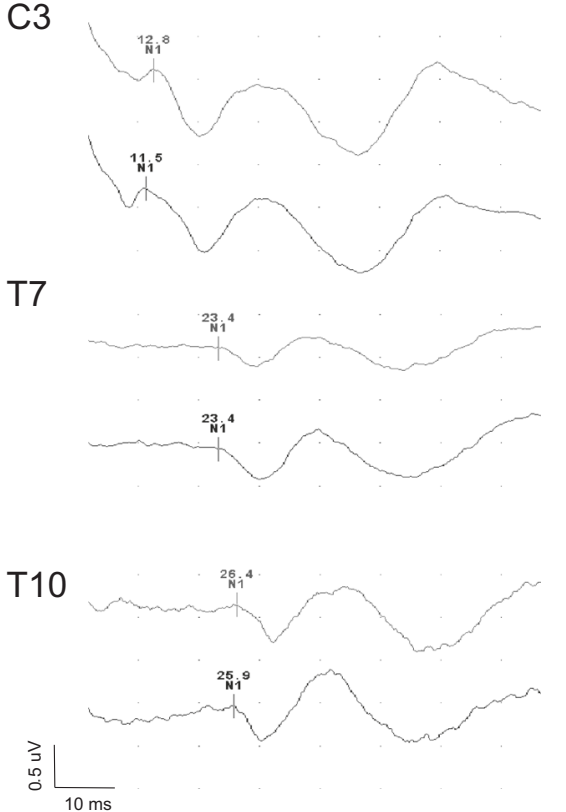

Fig. 3 CHEPs are sensitive to detect lesions within the anterior cord even when they are very focal, while they may not be detectable with SSEPs. This patient presents with a complete paralysis due to a spinalis anterior syndrome at level T7 (T2-weighted sagittal and axial MRI of the cord (a)). Thermal and pain sensation are lost below the level of lesion, while light touch is preserved. Accordingly CHEPs

for $9 \%$ of all SCI in a recent study of 839 individuals with SCI [39], upper extremity weakness dominates the clinical phenotype. The pathophysiological sequelae involved in CCS are complex. The central focus of spinal damage in combination with the special somatotopic organization of the descending tracts, where motor tracts for the upper limbs are localized more centrally than those for the lower extremities, were assumed to be responsible for the predominance of motor deficits in the hands in CSS. In addition, the traumatic impact on direct corticospinal (pyramidal) tracts, given their significant role in motor control of the hands and arms [40], may also play a fundamental role. In CCS, deficits in the pyramidal system along with concomitant cervical anterior horn damage can be detected by the combined use of MEPs and motor NCS (CMAPs, F-waves, and H-reflexes). CHEPs in conjunction with MEPs can be employed to characterize centromedullary cord damage, affecting the spinothalamic projections [32], while SSEPs remain normal as the dorsal cord remains unaffected (Fig. 3).

Besides the somatic nervous system, the autonomic nervous system (ANS) can also be evaluated in a topographical manner with electrophysiological testing in SCI. were abolished below T7 (b), while dSSEP remained normal (c). CHEPs at $\mathrm{T} 7$ are preserved due to the ascending Lissauer's tract terminating in the dorsal horn rostral to the lesion. For CHEPs an average of 15 stimuli and for dSSEP an average of 100 is presented for each stimulated dermatome. CHEP contact heat evoked potential, dSSEP dermatomal somatosensory evoked potential

This is, however, limited to the sympathetic system where sympathetic skin responses (SSRs) can be helpful to characterize the location of the lesion level and dysfunction of the spinal sympathetic intermedio-lateral cell columns [41]. SSRs may also serve to assess prognosis of potential severe complications such as autonomic dysreflexia in SCI [42]. However, to appreciate the full extent of ANS damage in SCI and to accomplish a full stratification for SCI trials, non-neurophysiological autonomic measures, such as heart rate variability, long term blood pressure assessments and quantitative testing of sweating, bowel, and bladder function should be considered.

Summary and Considerations: A gross idea about spinal tract deficits can be inferred from the ISNCSCI exam, however, electrophysiological testing helps to detect and quantify the extent and topography of spinal lesions and specific spinal syndromes. Based on the planned therapeutic interventions, it is helpful to know the severity of damage within specific spinal tracts rather than just the spinal cord as a whole. Topography of the spinal lesion is more important than a global classification of the injury as reflected in 
AIS grades. To this end, clinical assessments are complemented by electrophysiological measures such as motor responses (MEPs), sensorimotor segmental reflexes (H-reflex), and sensory assessments (SSEP/ CHEPs/LEPs) being specific to assess the integrity of segmental and longitudinal pathways.

Example: A characterization of a centro-medullary cord damage, affecting the spinothalamic projections, but not the dorsal columns is presented in Fig. 3.

\section{Lesion level and extent of lesion}

Traumatic SCI usually results in a diffuse damage zone of the spinal cord extending over 2-3 neurological spinal segments. This is reflected in the clinical description of a "zone of partial preservation" in sensorimotor complete (AIS A) SCI.

For the assessment of damage associated with a discrete spinal cord level, a segmental approach, involving a level-by-level assessment, is needed. Such a segmental approach crucially requires examining dermatomes above, at, and below the level of injury in well-defined regions of the body primarily innervated by individual spinal segments. Such segmental assessment can objectively be performed with CHEPs/LEPs, dermatomal SSEP and determination of segmental electrical perception thresholds (EPTs) [43, 44], as well as with EMG and MEP for certain lesion levels.

The assessment of EPT may add valuable information to clinical sensory testing in terms of localizing exact lesion level and as a standardized quantitative sensory readout. They are highly reproducible, reliable, and stable over the first 6 months after traumatic SCI [45]. EPT has been shown to be more sensitive in detecting the level of sensory perception impairment in SCI when compared with clinical testing with ISNCSCI. This can be explained due to the circumstance that a patient's impression of what constitutes normal sensation for a dermatome may be the result of his adaptation to a persistent deficit. This would result in a discrepancy between EPT and clinical assessments with a more reliable result derived from the objective perception thresholds [46].

MEPs can be used to stratify severity of the spinal lesion and evolution of motor function independent of AIS classification [12, 28]. Depending on the SCI lesion severity as assessed with transcranial magnetic stimulation, MEPs predicted motor recovery and ambulatory capacity $[26,28,46]$. In an approach with simultaneous recordings from multiple myotomes, MEPs could serve to objectively determine cervical lesion level [47]. However, MEPs are not useful measures to determine segmental damage at thoracic levels because even in complete SCI, MEP responses could be recorded from muscles at segments below the lesion as segmental innervation largely overlaps [48].

\begin{abstract}
Summary and Considerations: Sensory and motor lesion level can be confirmed using level-by-level electrophysiological assessments complementing the standard clinical ISNCSCI examination. Detection of altered EPT, MEP thresholds or dSSEP/CHEPs may indicate a change of lesion level. Objective measures of perception thresholds such as EPT, being more sensitive in the detection of a sensory level than clinical testing, may furthermore detect changes overlooked by clinical testing. Given the notion that effects of therapeutic-stimulated repair will likely be most readily observed in segments in close proximity to the lesion level [49] these segmental assessments become more valuable and important to determining meaningful clinical benefit.
\end{abstract}

Example: See Fig. 4 for an example of segmental assessment using dSSEP above, at, and below the level of lesion.

\section{Severity and completeness of $\mathrm{SCl}$}

The severity of SCI lesion has a strong impact on clinical recovery during rehabilitation and can be determined relatively early after the injury [1]. In clinical practice, the distinction between "complete" and "incomplete" SCI is commonly made to express the injury's severity. From a neurobiological perspective, however, this clinical classification must be considered to be relatively crude since a total anatomic transection of the spinal cord is uncommon (apart from some instances of penetrating trauma). By utilizing electrophysiological recordings, Dimitrijevic et al. coined the term "discomplete" SCI to describe clinically complete lesions, however, accompanied by neurophysiological evidence of residual brain influence on spinal motor activity $[18,50]$. According to their criteria, $84 \%$ of the participants with clinically complete SCI had a so-called motor discomplete SCI. Moreover, testing of SSEPs [15] and specific sensory modalities in AIS A patients using quantitative sensory testing [14] revealed sensory discomplete SCI. The presence of residual spinothalamic tract function has been proposed as an important determinant of below-level neuropathic pain following SCI [14].

MEPs and SSEPs can be used to stratify severity of the spinal lesion and evolution of motor function independent of AIS classification [12, 13, 28]. While the majority of patients who were clinically classified motor complete (AIS A and B) lacked MEPs in the leg muscles, there was 
Fig. 4 Segmental assessment of dorsal column integrity by dSSEP. The discrete level of a spinal cord lesion can objectively be determined and monitored very precisely employing a level-by-level assessment of the lesion (a). This patient suffered a complete (AIS A) traumatic cervical lesion at C6 (T2-weighted sagittal MRI of the cord (b)). dSSEP above the level of lesion are intact $(\mathrm{C} 4 / 5)$, while at and below the level of lesion dSSEPs are impaired (delayed EP at C6) or lost (C7/8), respectively. Electrical stimulations are applied at the key sensory points of each dermatome defined by the ISNCSCI. For each dSSEP an average of 100 stimuli is presented. dSSEP dermatomal somatosensory evoked potential a

dSSEP

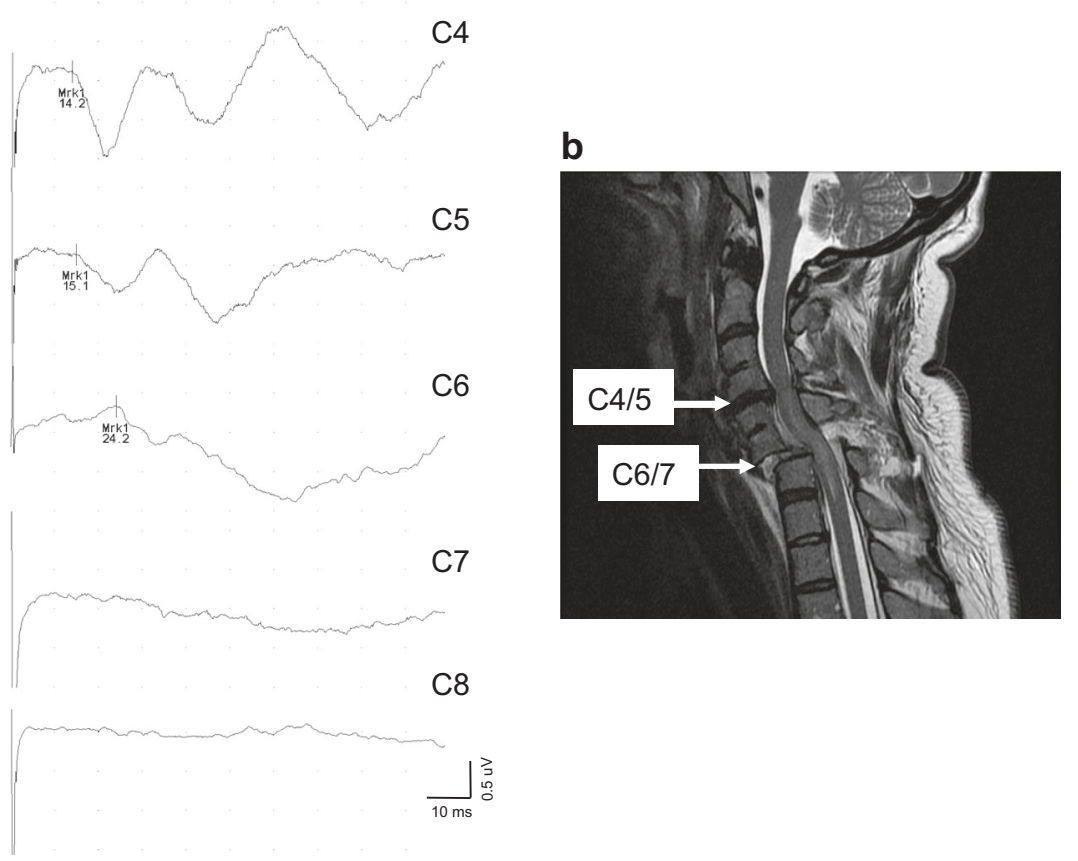

a small fraction of $2 \%$ who did show a small MEP during the course of recovery [28]. Likewise, there were 7\% positive upper extremity MEP responses despite a clinical motor-complete cervical injury [12]. This indicates that direct electrophysiological assessment with MEPs can reveal a subgroup of AIS A and B patients who do not present with complete cervical injury despite a clinical "complete" classification of AIS A. While upper extremity motor scores may yield a similar result when used as a criterion to detect discomplete cervical lesion, MEPs will be a more refined, objective, better scalable, and responsive measure of motor pathways, even in cases where patients are unable to recruit a muscle voluntarily (e.g., due to sensory deficits or noncompliance). Accordingly, depending on SCI severity as assessed with transcranial magnetic stimulation, the presence of MEPs correlates with motor recovery and potential for ambulatory capacity [26, 28, 46]. However, one drawback of transcranial magnetic stimulation over the motor cortex is the fact that it may not always be sufficiently strong to produce a supra-threshold descending volley and excite LMN, e.g., due to paucity of remaining descending fibers or deficits in synchronization within the lesioned cord.

The severity and completeness of SCI can vary across the somatic and autonomic spinal systems [49]. SSRs have been used to assess the completeness of autonomic disruption as has been shown in several studies so far [42, 51]. Although there is a clear trend for absent SSRs with higher and more severe sensorimotor completeness (based on an accompanying AIS classification), dissociation between sensorimotor and autonomic disruption (i.e., integrity of descending spinal autonomic pathways in the absence of descending motor pathways) has been reported [52]. Furthermore, abnormal SSR as a proxy for autonomic completeness has been associated with a sequelae of autonomic dysfunction in SCI, particularly autonomic dysreflexia and orthostatic hypotension (for review see [41]).

Summary and Considerations: Complete spinal lesions do not always present with a complete loss of evoked potentials, i.e., they are not all the same in their electrophysiological profile. This is found when stratifying SCI by means of sensory and motor evoked potentials independent from clinical assessment protocols $[12,13,28,53]$. Considering the gross nature of the ISNCSCI in its use to assess physiological completeness of SCI [11], it is recommended (where possible) to use functionally and neuroanatomically objective electrophysiological assessments in parallel with clinical testing when determining lesion severity and its evolution over time.

\section{Assessments of deterioration and/or adverse events}

A major concern of SCI clinical trials is neurological safety: will a therapy aimed at improving sensorimotor function 
inadvertently lead to neurological deterioration? Such an effect could occur in the course of therapeutic delivery (e.g., injection of cell-based therapies into the spinal cord), secondary to degenerative processes (e.g., increased secondary inflammation). For many of the same reasons that electrophysiology may play a pivotal role in the measurement of beneficial outcomes, they also may be important in the evaluation of trial safety.

The most pragmatic application of conventional electrophysiological testing in terms of safety relates to an objective assessment of the spinal lesion pattern and changes over time. Promoting neuroplasticity in the spinal cord represents a major target for acute and chronic clinical trials. To be effective, preclinical studies suggest that partial white matter sparing across the injury epicenter is a requisite starting point. In humans, this translates into trials primarily involving incomplete injuries. Based on the initial preservation of SSEPs and MEPs and their proven stability of latency over time [28, 53, 54], electrophysiological readouts, such as latency or an individual pattern of loss and preservation of potentials, may be useful as surrogate markers of detrimental effects (e.g., reduced amplitude and increased latencies) on connectivity through the lesion site.

In this regard, a thorough description of the natural evolution of SSEP and MEP data has been given in several studies presenting data from 200 to 300 patients during the first year following acute SCI [12, 13, 28, 53]. Next to a robust stratification of severity/completeness of SCI independent of clinical criteria (AIS), these studies show that most electrophysiological tests assessing spinal tracts show stable temporal indices (latency readouts) over time. For instance, MEP latencies remain stable (i.e., no trend toward normalization or deterioration) [28, 54], while MEP amplitudes increase during recovery from acute SCI $[28,55]$ and to some extent in chronic SCI subjects following functional training, such as weight-assisted treadmill training [56].

In individuals with more severe injuries (e.g., AIS A), however, such conductivity assessments of spinal tracts as SSEPs/CHEPs/MEPs (i.e., those where the signals traverse the lesion site) are limited in terms of evaluating safety as evoked potential across the lesion are likely not recordable and thus, no baseline recording may be obtained from which to measure a detrimental change (note: a beneficial effect can still be assessed). Of notable concern is the possibility that an intervention results in an ascending lesion (e.g., syringomyelia in individuals with AIS A SCI). This is a concern for both thoracic and cervical injuries, but would be particularly alarming in individuals with tetraplegia, potentially affecting remaining upper limb function, and activities of daily living [57]. To detect subtle deterioration near the level of injury (i.e., rostral and caudal), dSSEPs and CHEPs are useful in addition to conventional clinical assessments. Unlike MEPs, these sensory electrophysiological tools can be applied to test thoracic dermatomes $[44,58]$.

\begin{abstract}
Summary and Considerations: With regard to a timely and precise detection of adverse events, detection of altered EPT, MEP thresholds or dSSEP/CHEPs may indicate a change of lesion level. This might be indicative of adverse events or complications such as development of syringomyelia or maladaptive neuroplasticity (e.g., development of neuropathic pain).
\end{abstract}

Example: In a case with posttraumatic syringomyelia, i.e., a central syrinx affecting the crossing fibers in the anterior commissure, CHEPs were suited to detect the progression and spontaneous recovery of the disease [59] (see Fig. 5).

\section{Clinical trial outcome measures}

Currently, the majority of clinical trials in SCI are being conducted by industry interested in gaining approval for their therapeutic from regulatory agencies. Those regulatory agencies, in turn, usually request clinically relevant functional improvements as outcome measures. Unfortunately, this very high standard has rarely been met by clinical trials for neurological disorders. Electrophysiological methods can reveal a change of nerve function (i.e., natural recovery or treatment related) that might be overlooked by conventional clinical assessments. While it is possible that a positive therapeutic effect could be missed in an early phase clinical trial if functional improvement is made the primary outcome, this does not preclude that the intervention may have had a positive biological effect. It is crucial that such subclinical effects will be detected as they can hint that a therapeutic approach may reach clinical significance provided adequate modification, e.g., adjustment in how or in whom an intervention be applied or by improving the intervention itself. For example, if an intervention designed to enhance remyelination improved central conduction times, it might be worth further study after increasing its specific efficacy, even if, on the first pass, it failed to induce a clinically meaningful effect.

Detecting a positive biological effect of a given therapeutic in an electrophysiological assessment could mean proof-of-principle. Elucidating potential underlying mechanism of recovery may help the evolution of study designs in subsequent trial phases, so that eventually clinically meaningful outcome goals are achieved.

Upon completion of clinical trials, neurophysiological data can be useful to retrospectively identify features that distinguish responders from nonresponders and 


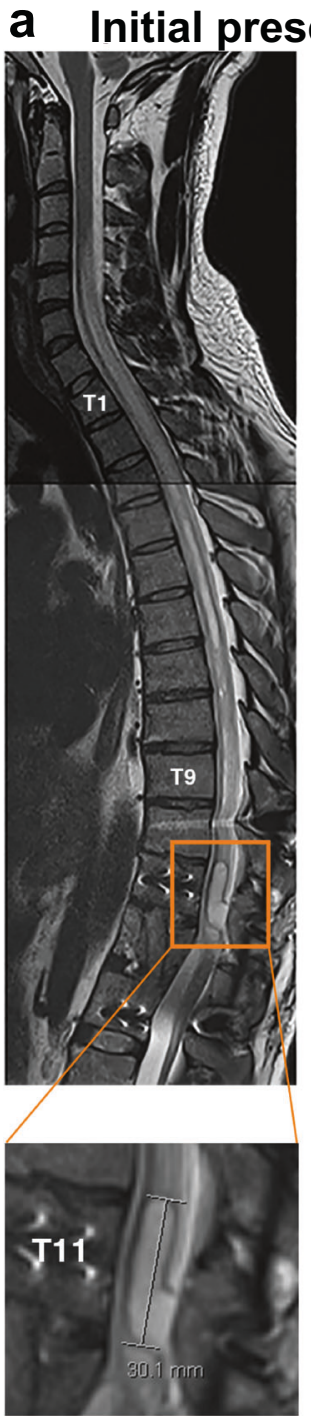

\section{b Month 2}
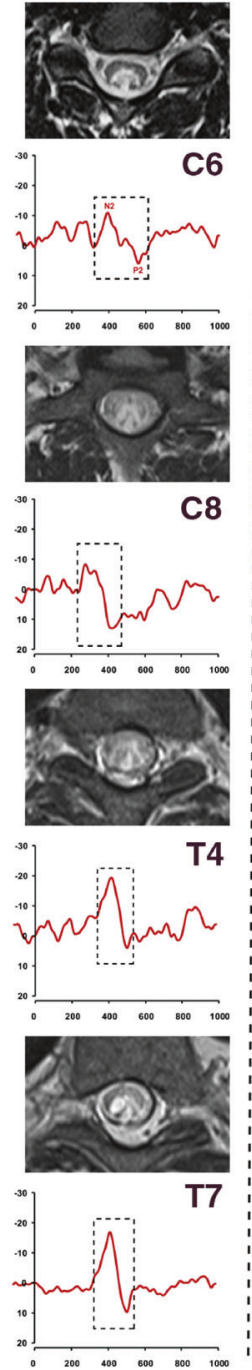

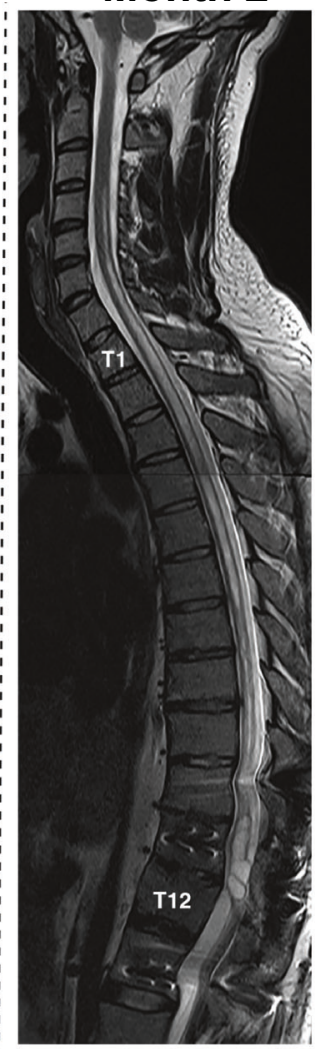

C Month 8
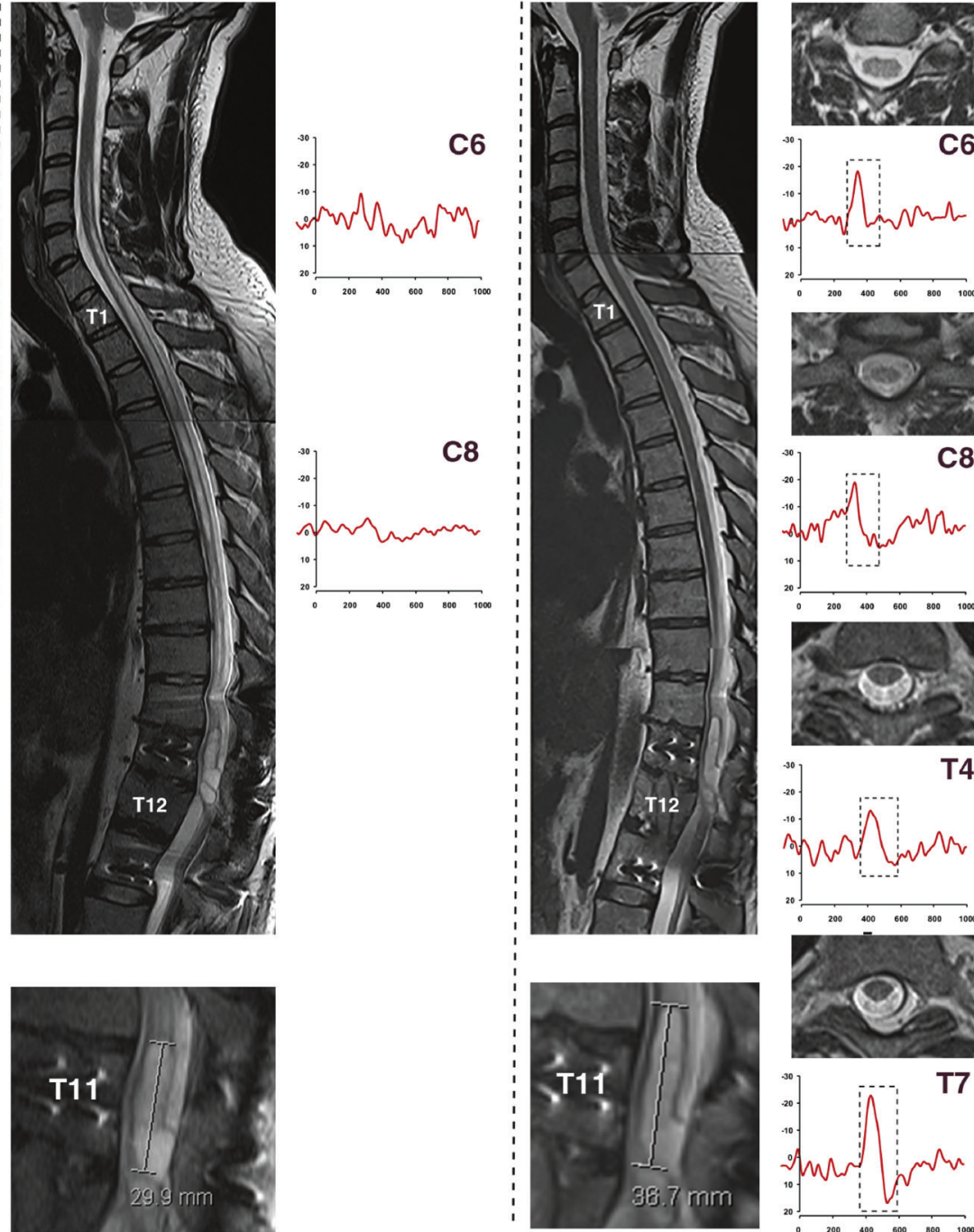
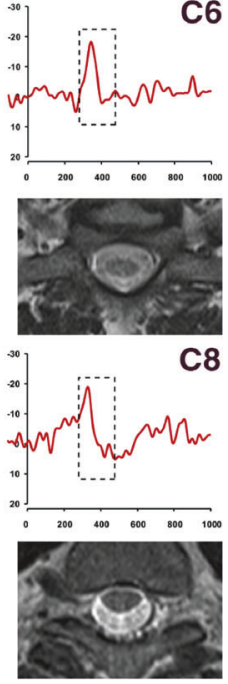

T4
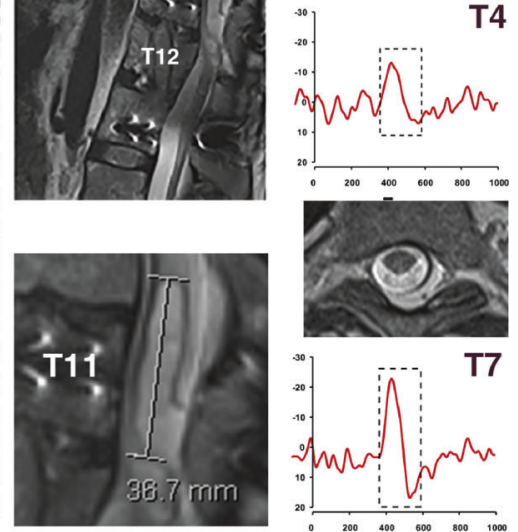

Fig. 5 Neuroimaging and electrophysiological assessment of a posttraumatic syrinx. T2-weighted images and CHEPs are shown at three time points (at initial presentation (a), 2 (b), and 8 months later (c)) in a 32-year-old patient with a chronic, incomplete (AIS C) SCI at T9 who presented with a symptomatic posttraumatic syrinx. Between initial presentation and 2 months, sagittal cervicothoracic MRI shows the development of the syrinx over several segments up to the cervical cord. Axial insets show the full extent of the syrinx cavity. The full development of the syrinx was associated with acute-onset of

consequently, inform patient stratification for future clinical trials. Taken together, the implementation of neurophysiological assessments may improve patient stratification, tracking treatment effects, and enhance the success rate of forthcoming clinical trials.

\section{General limitations of electrophysiology}

There are limitations to the application of electrophysiological studies. These type of studies require specific, costly neuropathic pain, impaired pinprick sensation, and abolished CHEPs from $\mathrm{C} 6$ and $\mathrm{C} 8$ dermatomes (b). At 8 months, the syrinx was resolved, symptoms reduced, and CHEPs as a likely marker of disease progression improved (c). CHEPs are ideally suited to assess syringomyelia preferentially affecting the spinothalamic crossing fibers in the anterior commissure. The resolution of the syrinx at month 8 was associated with a rostral extension of a preexisting, posttraumatic cyst at T11 (seen in the lowest panel). (Figure adapted from Killeen et al. [59])

equipment, as well as well-trained staff to guarantee reliable data acquisition with standardized recording configurations, testing conditions, protocols, and data processing/analysis. The effort of standardization of environmental circumstances, especially for multicenter trials, might limit the choice of participating centers to those with the requisite technical abilities and experience.

Classical electrophysiological assessments, such as SSEP, MEP, and NCS, test large fiber, highly myelinated neurons by applying artificial electrical or magnetic stimuli. Accordingly, electrophysiology is usually not directly 
related to functional outcome and thus, its correlation with integrity of spinal structures or function may be weak [60]. Assessing mainly large fiber function, electrophysiological measures test a small fraction of neural circuitry [61], and therefore, may overlook changes and adaptations subsequent to trauma to the CNS.

In order to guarantee high quality of electrophysiological tests and to bring them into routine use for clinical SCI trials, rigorous investigations on test quality, such as validity, reliability, as well as their sensitivity and responsiveness to change are needed.

\section{Conclusions}

The merit of including objective and quantitative measures of sensory, motor, and autonomic function, based on electrophysiological techniques, are addressed in this review. Future SCI trials implementing electrophysiology will likely benefit from improved SCI diagnosis and patient stratification, as well as detection of both beneficial and adverse events. Many of the above introduced electrophysiological assessments are nowadays routinely performed during spinal surgery, and thus, a growing number of centers are perfectly positioned to undertake these assessments outside the surgical suite. Electrophysiological methods can be rigorously standardized and harmonized protocols across clinical sites can improve data quality.

Funding Funding was provided by the American Spinal Injury Association (ASIA), Christopher and Dana Reeve Foundation (CDRF), Craig H. Neilsen Foundation (CHN), International Research Foundation for Paraplegia (IRP), Rick Hansen Institute (RHI), Spinal Cord Outcomes Partnership Endeavor (SCOPE), Spinal Research (SR), Wings for Life (WfL).

Author Contributions $\mathrm{MH}$ was responsible for manuscript assembly, conducting the search, compiling the patient cases, creating the Table and writing the manuscript. MS was responsible for conducting research, compiling the patient cases and writing the manuscript. All remaining authors (JLKK, CRJ, JR, JCF, and KET) contributed individually by conducting the search and writing individual sections of the manuscript.

\section{Compliance with ethical standards}

Conflict of interest The authors declare that they have no conflict of interest.

Ethical approval We certify that all applicable institutional and governmental regulations concerning the ethical use of human volunteers were followed during the data collection for patient examples provided in this manuscript. The local ethics board 'Kantonale Ethikkommission Zürich, KEK' (EK-04/2006, PB_2016-02051, clinicaltrial.gov number: NCT02138344) has approved this data collection.

Publisher's note: Springer Nature remains neutral with regard to jurisdictional claims in published maps and institutional affiliations.

\section{References}

1. Fawcett JW, Curt A, Steeves JD, Coleman WP, Tuszynski MH, Lammertse D, et al. Guidelines for the conduct of clinical trials for spinal cord injury as developed by the ICCP panel: spontaneous recovery after spinal cord injury and statistical power needed for therapeutic clinical trials. Spinal Cord. 2007;45:190-205.

2. Steeves JD, Lammertse D, Curt A, Fawcett JW, Tuszynski MH, Ditunno JF, et al. Guidelines for the conduct of clinical trials for spinal cord injury (SCI) as developed by the ICCP panel: clinical trial outcome measures. Spinal Cord. 2007;45:206-21.

3. Lammertse D, Tuszynski MH, Steeves JD, Curt A, Fawcett JW, Rask C, et al. Guidelines for the conduct of clinical trials for spinal cord injury as developed by the ICCP panel: clinical trial design. Spinal Cord. 2007;45:232-42.

4. Tuszynski MH, Steeves JD, Fawcett JW, Lammertse D, Kalichman M, Rask C, et al. Guidelines for the conduct of clinical trials for spinal cord injury as developed by the ICCP panel: clinical trial inclusion/exclusion criteria and ethics. Spinal Cord. 2007;45:222-31.

5. Furlan JC, Fehlings MG, Tator CH, Davis AM. Motor and sensory assessment of patients in clinical trials for pharmacological therapy of acute spinal cord injury: psychometric properties of the ASIA standards. J Neurotrauma. 2008;25:1273-301.

6. Alexander MS, Anderson KD, Biering-Sorensen F, Blight AR, Brannon R, Bryce TN, et al. Outcome measures in spinal cord injury: recent assessments and recommendations for future directions. Spinal Cord. 2009;47:582-91.

7. Krassioukov A, Biering-Sorensen F, Donovan W, Kennelly M, Kirshblum S, Krogh K, et al. International standards to document remaining autonomic function after spinal cord injury. J Spinal Cord Med. 2012;35:201-10.

8. Tanadini LG, Steeves JD, Hothorn T, Abel R, Maier D, Schubert $\mathrm{M}$, et al. Identifying homogeneous subgroups in neurological disorders: unbiased recursive partitioning in cervical complete spinal cord injury. Neurorehabilit Neural Repair. 2014;28:507-15.

9. Cadotte DW, Fehlings MG. Will imaging biomarkers transform spinal cord injury trials? Lancet Neurol. 2013;12:843-4.

10. Hupp M, Pavese C, Bachmann L, Koller R, Schubert M. Electrophysiological multimodal assessments improve outcome prediction in traumatic cervical spinal cord injury. J Neurotrauma. 2018;35:2916-23.

11. Spiess MR, Muller RM, Rupp R, Schuld C, Group E-SS, van Hedel HJ. Conversion in ASIA impairment scale during the first year after traumatic spinal cord injury. $\mathrm{J}$ Neurotrauma. 2009;26:2027-36.

12. Petersen JA, Spiess M, Curt A, Weidner N, Rupp R, Abel R, et al. Upper limb recovery in spinal cord injury: involvement of central and peripheral motor pathways. Neurorehabilit Neural Repair. 2017;31:432-41.

13. Spiess M, Schubert M, Kliesch U, group E-SS, Halder P. Evolution of tibial SSEP after traumatic spinal cord injury: baseline for clinical trials. Clin Neurophysiol. 2008;119:1051-61.

14. Wasner G, Lee BB, Engel S, McLachlan E. Residual spinothalamic tract pathways predict development of central pain after spinal cord injury. Brain. 2008;131:2387-400.

15. Finnerup NB, Gyldensted C, Fuglsang-Frederiksen A, Bach FW, Jensen TS. Sensory perception in complete spinal cord injury. Acta Neurol Scand. 2004;109:194-9.

16. Biering-Sorensen F, Alai S, Anderson K, Charlifue S, Chen Y, DeVivo $\mathrm{M}$, et al. Common data elements for spinal cord injury clinical research: a National Institute for Neurological Disorders and Stroke project. Spinal Cord. 2015;53:265-77.

17. Kirshblum SC, Burns SP, Biering-Sorensen F, Donovan W, Graves DE, Jha A, et al. International standards for neurological 
classification of spinal cord injury (revised 2011). J Spinal Cord Med. 2011;34:535-46.

18. McKay WB, Lim HK, Priebe MM, Stokic DS, Sherwood AM. Clinical neurophysiological assessment of residual motor control in post-spinal cord injury paralysis. Neurorehabilit Neural Repair. 2004;18:144-53.

19. Curt A, Dietz V. Neurographic assessment of intramedullary motoneurone lesions in cervical spinal cord injury: consequences for hand function. Spinal Cord. 1996;34:326-32.

20. Machida M, Yamada T, Ross M, Kimura J, Hitchon P. Effect of spinal cord ischemia on compound muscle action potentials and spinal evoked potentials following spinal cord stimulation in the dog. J Spinal Disord. 1990;3:345-52.

21. Oeda T, Kanda M, Onishi K, Kaji R, Akiguchi I. The value of Fwaves in an early electrodiagnosis of the lower cervical cord infarction. Rinsho Shinkeigaku. 1996;36:587-9.

22. Van De Meent H, Hosman AJ, Hendriks J, Zwarts M, Schubert M. Severe degeneration of peripheral motor axons after spinal cord injury: a European multicenter study in 345 patients. Neurorehabilit Neural Repair. 2010;24:657-65.

23. Boland RA, Lin CS, Engel S, Kiernan MC. Adaptation of motor function after spinal cord injury: novel insights into spinal shock. Brain. 2011;134:495-505.

24. Lin CS, Macefield VG, Elam M, Wallin BG, Engel S, Kiernan MC. Axonal changes in spinal cord injured patients distal to the site of injury. Brain. 2007;130:985-94.

25. Parry GJ. Electrodiagnostic studies in the evaluation of peripheral nerve and brachial plexus injuries. Neurol Clin. 1992;10:921-34.

26. Curt A, Dietz V. Electrophysiological recordings in patients with spinal cord injury: significance for predicting outcome. Spinal Cord1999;37:157-65.

27. Petersen JA, Schubert M, Dietz V. The occurrence of the Babinski sign in complete spinal cord injury. J Neurol. 2010;257:38-43.

28. Petersen JA, Spiess M, Curt A, Dietz V, Schubert M. Spinal cord injury: one-year evolution of motor-evoked potentials and recovery of leg motor function in 255 patients. Neurorehabilit Neural Repair. 2012;26:939-48.

29. Little JW, Halar E. Temporal course of motor recovery after Brown-Sequard spinal cord injuries. Paraplegia. 1985;23:39-46.

30. Haefeli J, Kramer JL, Blum J, Curt A. Assessment of spinothalamic tract function beyond pinprick in spinal cord lesions: a contact heat evoked potential study. Neurorehabilit Neural Repair. 2014;28:494-503.

31. Yamada T. Neuroanatomic substrates of lower extremity somatosensory evoked potentials. J Clin Neurophysiol. 2000;17:269-79.

32. Ulrich A, Haefeli J, Blum J, Min K, Curt A. Improved diagnosis of spinal cord disorders with contact heat evoked potentials. Neurology. 2013;80:1393-9.

33. Ulrich A, Min K, Curt A. High sensitivity of contact-heat evoked potentials in "snake-eye" appearance myelopathy. Clin Neurophysiol. 2015;126:1994-2003.

34. Fisher KM, Zaaimi B, Baker SN. Reticular formation responses to magnetic brain stimulation of primary motor cortex. J Physiol. 2012;590:4045-60.

35. Matsugi A, Mori N, Uehara S, Kamata N, Oku K, Okada Y. et al. Effect of cerebellar transcranial magnetic stimulation on soleus Ia presynaptic and reciprocal inhibition. Neuroreport.2015;26:139-43.

36. Di Lazzaro V, Pilato F, Oliviero A, Saturno E, Dileone M, Tonali PA. Role of motor evoked potentials in diagnosis of cauda equina and lumbosacral cord lesions. Neurology. 2004;63:2266-71.

37. Schneider RC. The syndrome of acute anterior spinal cord injury. J Neurosurg. 1955;12:95-122.

38. Bromm B, Frieling A, Lankers J. Laser-evoked brain potentials in patients with dissociated loss of pain and temperature sensibility. Electroencephalogr Clin Neurophysiol. 1991;80:284-91.
39. Molliqaj G, Payer M, Schaller K, Tessitore E. Acute traumatic central cord syndrome: a comprehensive review. Neurochirurgie. 2014;60:5-11.

40. Lemon RN, Kirkwood PA, Maier MA, Nakajima K, Nathan P. Direct and indirect pathways for corticospinal control of upper limb motoneurons in the primate. Prog Brain Res. 2004;143:263-79.

41. Berger MJ, Hubli M, Krassioukov AV. Sympathetic skin responses and autonomic dysfunction in spinal cord injury. J Neurotrauma. 2014;31:1531-9.

42. Curt A, Weinhardt C, Dietz V. Significance of sympathetic skin response in the assessment of autonomic failure in patients with spinal cord injury. J Auton Nerv Syst 1996;61:175-80.

43. Kramer JK, Taylor P, Steeves JD, Curt A. Dermatomal somatosensory evoked potentials and electrical perception thresholds during recovery from cervical spinal cord injury. Neurorehabilit Neural Repair 2010;24:309-17.

44. Jutzeler CR, Rosner J, Rinert J, Kramer JL, Curt A. Normative data for the segmental acquisition of contact heat evoked potentials in cervical dermatomes. Sci Rep. 2016;6:34660.

45. van Hedel HJ, Kumru H, Rohrich F, Galen S. Changes in electrical perception threshold within the first 6 months after traumatic spinal cord injury: a multicenter responsiveness study. Neurorehabilit Neural Repair. 2012;26:497-506.

46. Ellaway PH, Kuppuswamy A, Balasubramaniam AV, Maksimovic R, Gall A, Craggs MD, et al. Development of quantitative and sensitive assessments of physiological and functional outcome during recovery from spinal cord injury: a clinical initiative. Brain Res Bull. 2011;84:343-57.

47. Shields CB, Ping Zhang Y, Shields LB, Burke DA, Glassman SD. Objective assessment of cervical spinal cord injury levels by transcranial magnetic motor-evoked potentials. Surg Neurol. 2006;66:475-83. discussion 483.

48. Cariga P, Catley M, Nowicky AV, Savic G, Ellaway PH, Davey NJ. Segmental recording of cortical motor evoked potentials from thoracic paravertebral myotomes in complete spinal cord injury. Spine. 2002;27:1438-43.

49. Ellaway PH, Anand P, Bergstrom EM, Catley M, Davey NJ, Frankel HL, et al. Towards improved clinical and physiological assessments of recovery in spinal cord injury: a clinical initiative. Spinal Cord. 2004;42:325-37.

50. Dimitrijevic MR. Residual motor functions in spinal cord injury. Adv Neurol. 1988;47:138-55.

51. Cariga P, Catley M, Mathias CJ, Savic G, Frankel HL, Ellaway $\mathrm{PH}$. Organisation of the sympathetic skin response in spinal cord injury. J Neurol Neurosurg Psychiatry. 2002;72:356-60.

52. Claydon VE, Krassioukov AV. Orthostatic hypotension and autonomic pathways after spinal cord injury. J Neurotrauma. 2006;23:1713-25.

53. Kuhn F, Halder P, Spiess MR, Schubert M, Group E-SS. One-year evolution of ulnar somatosensory potentials after trauma in 365 tetraplegic patients: early prediction of potential upper limb function. J Neurotrauma. 2012;29:1829-37.

54. Wirth B, Van Hedel HJ, Curt A. Changes in corticospinal function and ankle motor control during recovery from incomplete spinal cord injury. J Neurotrauma. 2008;25:467-78.

55. Wirth B, van Hedel HJ, Curt A. Ankle dexterity is less impaired than muscle strength in incomplete spinal cord lesion. J Neurol. 2008;255:273-9.

56. Thomas SL, Gorassini MA. Increases in corticospinal tract function by treadmill training after incomplete spinal cord injury. $\mathrm{J}$ Neurophysiol. 2005;94:2844-55.

57. Kramer JL, Lammertse DP, Schubert M, Curt A, Steeves JD. Relationship between motor recovery and independence after sensorimotor-complete cervical spinal cord injury. Neurorehabilit Neural Repair. 2012;26:1064-71. 
58. Slimp JC, Rubner DE, Snowden ML, Stolov WC. Dermatomal somatosensory evoked potentials: cervical, thoracic, and lumbosacral levels. Electroencephalogr Clin Neurophysiol. 1992; 84:55-70.

59. Killeen T, Rosner J, Jutzeler CR, Hupp M, Heilbronner R, Curt A. Spontaneous resolution of an extensive posttraumatic syrinx. Neurology. 2016;87:1299-301.

60. Wirth B, van Hedel HJ, Curt A. Ankle paresis in incomplete spinal cord injury: relation to corticospinal conductivity and ambulatory capacity. J Clin Neurophysiol. 2008;25:210-7.

61. Firmin L, Field P, Maier MA, Kraskov A, Kirkwood PA, Nakajima $\mathrm{K}$, et al. Axon diameters and conduction velocities in the macaque pyramidal tract. J Neurophysiol. 2014;112:1229-40.

62. Kohara N, Kimura J, Kaji R, Goto Y, Ishii J, Takiguchi M, et al. F-wave latency serves as the most reproducible measure in nerve conduction studies of diabetic polyneuropathy: multicentre analysis in healthy subjects and patients with diabetic polyneuropathy. Diabetologia. 2000;43:915-21.

63. Pinheiro DS, Manzano GM, Nobrega JA. Reproducibility in nerve conduction studies and F-wave analysis. Clin Neurophysiol. 2008;119:2070-3.

64. Pauri F, Boffa L, Cassetta E, Pasqualetti P, Rossini PM. Botulinum toxin type-A treatment in spastic paraparesis: a neurophysiological study. J Neurol Sci. 2000;181:89-97.

65. Hiersemenzel LP, Curt A, Dietz V. From spinal shock to spasticity: neuronal adaptations to a spinal cord injury. Neurology. 2000;54:1574-82.

66. (AAEM) AAoEM. The electrodiagnostic evaluation of patients with suspected cervical radiculopathy: literature review on the usefulness of needle electromyography. Muscle Nerve. 1999;22: S213-21.

67. Narayanaswami P, Geisbush T, Jones L, Weiss M, Mozaffar T, Gronseth G, et al. Critically re-evaluating a common technique: accuracy, reliability, and confirmation bias of EMG. Neurology. 2016;86:218-23.

68. Calder KM, Stashuk DW, McLean L. Motor unit potential morphology differences in individuals with non-specific arm pain and lateral epicondylitis. J Neuroeng Rehabil 2008;5:34.

69. Takehara I, Chu J, Li TC, Schwartz I. Reliability of quantitative motor unit action potential parameters. Muscle Nerve. 2004;30:111-3.

70. Palmieri RM, Hoffman MA, Ingersoll CD. Intersession reliability for H-reflex measurements arising from the soleus, peroneal, and tibialis anterior musculature. Int J Neurosci. 2002;112:841-50.

71. Hopkins JT, Ingersoll CD, Cordova ML, Edwards JE. Intrasession and intersession reliability of the soleus H-reflex in supine and standing positions. Electroencephalogr Clin Neurophysiol. 2000;40:89-94.

72. Hopkins JT, Wagie NC. Intrasession and intersession reliability of the quadriceps Hoffmann reflex. Electroencephalogr Clin Neurophysiol. 2003;43:85-9.

73. Phadke CP, Thompson FJ, Trimble MH, Behrman AL, Kukulka CG. Reliability of soleus H-reflexes in standing and walking postincomplete spinal cord injury. Int J Neurosci. 2010;120:128-36.
74. Curt A, Dietz V. Traumatic cervical spinal cord injury: relation between somatosensory evoked potentials, neurological deficit, and hand function. Arch Phys Med Rehabil. 1996;77:48-53.

75. Curt A, Dietz V. Ambulatory capacity in spinal cord injury: significance of somatosensory evoked potentials and ASIA protocol in predicting outcome. Arch Phys Med Rehabil. 1997;78:39-43.

76. Jutzeler CR, Streijger F, Aguilar J, Shortt K, Manouchehri N, Okon E, et al. Sensorimotor plasticity after spinal cord injury: a longitudinal and translational study. Ann Clin Transl Neurol. 2019;6:68-82.

77. Kramer JL, Moss AJ, Taylor P, Curt A. Assessment of posterior spinal cord function with electrical perception threshold in spinal cord injury. J Neurotrauma. 2008;25:1019-26.

78. Savic G, Bergstrom EM, Frankel HL, Jamous MA, Ellaway PH, Davey NJ. Perceptual threshold to cutaneous electrical stimulation in patients with spinal cord injury. Spinal Cord. 2006;44:560-6.

79. Leong GW, Gorrie CA, Ng K, Rutkowski S, Waite PM. Electrical perceptual threshold testing: a validation study. J Spinal Cord Med. 2009;32:140-6.

80. Lagerburg V, Bakkers M, Bouwhuis A, Hoeijmakers JG, Smit AM, Van Den Berg SJ, et al. Contact heat evoked potentials: normal values and use in small-fiber neuropathy. Muscle Nerve. 2015;51:743-9.

81. Rosner J, Hubli M, Hostettler P, Scheuren PS, Rinert J, Kramer JLK, et al. Contact heat evoked potentials: Reliable acquisition from lower extremities. Clin Neurophysiol. 2018;129:584-91.

82. Kramer JL, Haefeli J, Curt A, Steeves JD. Increased baseline temperature improves the acquisition of contact heat evoked potentials after spinal cord injury. Clin Neurophysiol. 2012;123:582-9.

83. Jutzeler CR, Ulrich A, Huber B, Rosner J, Kramer JLK, Curt A. Improved diagnosis of cervical spondylotic myelopathy with contact heat evoked potentials. J Neurotrauma. 2017;34:2045-53.

84. Treede RD, Lankers J, Frieling A, Zangemeister WH, Kunze K, Bromm B. Cerebral potentials evoked by painful, laser stimuli in patients with syringomyelia. Brain. 1991;114:1595-607. (Pt 4)

85. Iannetti GD, Truini A, Galeotti F, Romaniello A, Manfredi M, Cruccu G. Usefulness of dorsal laser evoked potentials in patients with spinal cord damage: report of two cases. J Neurol Neurosurg Psychiatry. 2001;71:792-4.

86. Potter-Baker KA, Janini DP, Frost FS, Chabra P, Varnerin N, Cunningham DA, et al. Reliability of TMS metrics in patients with chronic incomplete spinal cord injury. Spinal Cord. 2016;54:980-90.

87. Pellegrini M, Zoghi M, Jaberzadeh S. The effect of transcranial magnetic stimulation test intensity on the amplitude, variability and reliability of motor evoked potentials. Brain Res. 2018;1700:190-8.

88. van Hedel HJ, Murer C, Dietz V, Curt A. The amplitude of lower leg motor evoked potentials is a reliable measure when controlled for torque and motor task. J Neurol. 2007;254:1089-98.

89. Hubli M, Krassioukov AV. How reliable are sympathetic skin responses in subjects with spinal cord injury? Clin Auton Res. 2015;25:117-24.

90. Dietz V, Curt A. Neurological aspects of spinal-cord repair: promises and challenges. Lancet Neurol. 2006;5:688-94. 